\title{
Nogo-B promotes the epithelial-mesenchymal transition in HeLa cervical cancer cells via Fibulin-5
}

\author{
WEI XIAO ${ }^{1}$, SHUMIN ZHOU ${ }^{1}$, HUA XU $^{2}$, HENG LI $^{2}$, GUOQING HE $^{1}$, YINGLE LIU $^{1}$ and YIPENG QI ${ }^{1}$ \\ ${ }^{1}$ State Key Laboratory of Virology, College of Life Sciences, Wuhan University; ${ }^{2}$ Department of Urology, \\ Tongji Hospital, Tongji Medical College, Huazhong University of Science and Technology, Hubei, Wuhan, P.R. China
}

Received May 21, 2012; Accepted August 30, 2012

DOI: $10.3892 /$ or.2012.2069

\begin{abstract}
Cervical cancer is a common malignancy in women worldwide, and the occurrence of invasion and metastasis is the major cause for most cancer-related deaths. Epithelialmesenchymal transition (EMT) has been implicated in the metastasis of primary tumors and provides molecular mechanisms for cervical cancer metastasis. We previously reported that Nogo-B mediates cell motility by binding Fibulin-5. Herein, we show that the increased expression of Nogo-B is correlated with the degree of cervical cancer metastasis. In HeLa cervical cancer cells, overexpression of Nogo-B induces the EMT and promotes cell migration and invasion, while inhibiting cell adhesion. Furthermore, we found that Nogo-B accumulates and co-localizes with Fibulin-5 in pseudopods, and the downstream effects of overexpression of Nogo-B on cell motility could be partially abolished by RNA interference against Fibulin-5. These results suggest that Nogo-B functions as an inducer of cervical cancer metastasis and that this effect is mediated, at least in part, through Fibulin-5.
\end{abstract}

\section{Introduction}

Cervical carcinoma is the second most prevalent female cancer worldwide. Despite the generally good prognosis for early-stage cervical cancer patients, many patients still die as a result of metastasis and recurrence (1). How cervical cancer cells acquire the ability to invade surrounding tissue is unclear, but epithelial-mesenchymal transition (EMT) likely plays a role (2). Unfortunately, little information is available on the regulation of EMT in cervical cancer cells and on the association of EMT program with clinical outcome of cervical cancer patients.

EMT is an intricate process by which epithelial cells lose their epithelial characteristics and acquire a mesenchymal-like

Correspondence to: Professor Yipeng Qi, State Key Laboratory of Virology, College of Life Sciences, Wuhan University, Hubei, Wuhan 430072, P.R. China

E-mail: qiyipeng@whu.edu.cn

Key words: Nogo-B, Fibulin-5, epithelial-mesenchymal transition, cervical cancer metastasis phenotype (3). EMT was originally described in early embryogenesis as an essential process for specific developmental stages that require migration and transient dedifferentiation of embryonic epithelial cells (4). In recent years, EMT has been described as a relevant process in tumor progression and is not only implicated in tumor invasion but also in other stages during metastasis and in therapeutic resistance (5).

Nogo isoforms (Nogo-A, -B and -C) belong to the reticulum super family of proteins (6). These three isoforms share a conserved reticulum homology domain (RHD), which contains a 66-aa loop domain termed Nogo-66 (7). Nogo-B/ASY is widely expressed in many cell types and was originally characterized as a novel human apoptosis-inducing protein (8). Nogo-B/ ASY overexpression induces apoptosis through ER stress and ER-specific signaling pathways (9). Recent studies have shown that Nogo-B plays a role in cell adhesion and migration $(7,10,11)$; the amino terminus of Nogo-B (AmNogo-B) promotes the adhesion and migration of endothelial cells and negatively regulates platelet-derived growth factor-induced migration in smooth muscle cells (11).

Fibulin-5 is a recently identified fibulin family member (12). Distinguished from other fibulins, Fibulin-5 contains a conserved RGD motif that binds to integrins and mediates endothelial cell adhesion $(13,14)$. Fibulin-5 can also suppress angiogenesis in an RGD-dependent manner (15).

We previously reported that Nogo-B binds to Fibulin-5 both in vitro and in vivo. In this study, using HeLa cells as a model, we found that overexpression of Nogo-B promotes epithelial-mesenchymal transitions in a Fibulin-5-dependent manner. These results may contribute to a better understanding the function of Nogo-B in tumor cell migration and cancer metastasis.

\section{Materials and methods}

Patients and samples. We collected a total of 55 tissue specimens, including 14 normal cervical tissues, 10 cervical intraepithelial neoplasia (CIN) tissues, 31 cervical cancer tissues between September 2010 and December 2011 in Tongji Hospital, Tongji Medical College, Huazhong University of Science and Technology. Normal cervical tissues were derived from the patients who underwent hysterectomy due to nonmalignant disease. Cervical cancer tissues were obtained from primary untreated patients under colposcopy. Tumor stage 
Table I. Clinicopathological data for 55 patients providing cervical tissues.

\begin{tabular}{lccc}
\hline Characteristic & $\begin{array}{c}\text { Cancer } \\
(\mathrm{n}=31)\end{array}$ & $\begin{array}{c}\text { CIN } \\
(\mathrm{n}=10)\end{array}$ & $\begin{array}{c}\text { Normal } \\
(\mathrm{n}=14)\end{array}$ \\
\hline Age (mean \pm SD) & $47 \pm 6.2$ & $39 \pm 3.6$ & $41 \pm 5.9$ \\
Grade & & & \\
G1 & 11 & & \\
G2 & 12 & & \\
G3 & 8 & & \\
Stage & & & \\
I-II & 19 & & \\
III-IV & 12 & & \\
HPV infection & & & \\
+ & 31 & 9 & \\
- & 0 & 1 & \\
CIN classification & & & \\
CIN1 & & 3 & \\
CIN2-3 & & 7 & \\
\hline
\end{tabular}

and grade were determined according to the International Federation of Gynecology and Obstetrics standards (FIGO). Clinicopathological data for these patients are shown in Table I. Informed consents were obtained from all the patients.

This study was approved by the Ethics Committee of Tongji Hospital, Tongji Medical College, Huazhong University of Science and Technology. All the samples were immediately snap-frozen and stored in liquid nitrogen $\left(-180^{\circ} \mathrm{C}\right)$. To obtain homogeneous and histological well-characterized samples for RNA analyses, the nature of the tissue and its specified composition were determined by an experienced pathologist. All cancer samples contained at least $80 \%$ tumor tissues without necrosis. Normal cervical samples were verified to be free of any cervical lesions. CIN samples were verified to contain at least $80 \%$ CIN tissues.

Antibodies and reagents. Anti-Nogo-B, anti-Fibulin-5 and anti$\beta$-actin antibodies, horseradish peroxidase-conjugated anti-goat and anti-rabbit IgG antibodies, Cy3-conjugated goat anti-rabbit IgG antibodies and FITC-conjugated rabbit anti-goat IgG antibodies were purchased from Santa Cruz Biotechnology, Inc. Anti-E-cadherin, anti-N-cadherin and anti-vimentin antibodies were purchased from Cell Signaling Technology.

Real-time PCR. Total RNA from tissues and cells was isolated by using the AxyPrep Multisource Total RNA miniprep kit (Axygen) and reverse transcribed by PrimeScript RT Master Mix Perfect Real-time (Takara). The resulting cDNA samples were amplified by real-time PCR using gene-specific primer sets in conjunction with the SYBR Premix Ex Taq (Takara) in an Mx3000P instrument. The qPCR was performed with the following conditions: activation at $95^{\circ} \mathrm{C}$ for $5 \mathrm{~min}$ followed by 40 cycles of denaturation at $94^{\circ} \mathrm{C}$ for $15 \mathrm{sec}$, amplification at $60^{\circ} \mathrm{C}$ for $30 \mathrm{sec}$, elongation at $72^{\circ} \mathrm{C}$ for $30 \mathrm{sec}$. In the last, a cycle of solubility curve was added to examine the amplification quality. For all RT-PCR analyses, GAPDH mRNA was used to normalize the RNA inputs. The primer sequences used to amplify the genes are listed in Table II.

Cell culture and transfection. Human cervix epithelial adenocarcinoma HeLa cells were cultured in a humidified $5 \% \mathrm{CO}_{2}$ incubator at $37^{\circ} \mathrm{C}$ in DMEM supplemented with $10 \%$ fetal bovine serum (FBS). DNA transfections were performed using the FuGene HD Transfection Reagent (Roche), and RNA transfections were performed using the X-tremeGene siRNA Transfection Reagent (Roche). Stable HeLa cells overexpressing Nogo-B were constructed by transfection and selected with puromycin. HeLa cells stably overexpressing the empty vector were used as a negative control and are referred to as Blank.

RNA interference. The pSilencer 3.0 recombinant vectors were constructed by inserting 64-mer synthetic DNA oligonucleotides that encode two 19-nt reverse complements with homology to a portion of the target gene. For the three Nogo-B siRNA constructs, siA1: (5'-GTTTGCAGTGTTGATGTGG-3'), siA2: (5'-GTCCCTGGATTGAAGCGCAA-3') and siA3: (5'-ATCAGATGAAGGCCACCCA-3'), the respective target sequences are nt 903-921, nt 1092-1110 and nt 741-759. The siRNAs against Fibulin-5 (sc-43121) were purchased from Santa Cruz Biotechnology, Inc. and a scrambled siRNA was used as a control.

Immunofluorescence. HeLa cells were fixed in $4 \%$ paraformaldehyde for $15 \mathrm{~min}$ at $25^{\circ} \mathrm{C}$, followed by permeabilization in $1 \%$ Triton X-100. The cells were then washed with PBS and blocked with $0.5 \% \mathrm{BSA}$ for $1 \mathrm{~h}$ at $25^{\circ} \mathrm{C}$. Then, the cells were incubated with primary antibody at $4^{\circ} \mathrm{C}$ overnight, washed three times with PBS, incubated with secondary antibody for $45 \mathrm{~min}$ at room temperature, washed five times with PBS and mounted on a slide with $50 \%$ glycerol. Confocal images were captured with a Leica TCS SP2 AOBS MP.

In vitro cell migration and invasion assays. Cell invasion and migration assays were performed as described (16). Briefly, the cells were starved with serum-free DMEM for $24 \mathrm{~h}$ before performing the assays, and the cells were then added in DMEM with $0.1 \%$ FBS to the upper chamber of a Transwell plate (Costar) containing a membrane with $8-\mu \mathrm{m}$ pores. For the cell migration assays, the cells were allowed to migrate for $12 \mathrm{~h}$ into the lower chamber containing DMEM with $10 \%$ FBS. The cell invasion assays were similarly performed, except that cells were cultured on inserts with Matrigel (BD Biosciences) and allowed to invade for $24 \mathrm{~h}$. Finally, the cells that invaded the synthetic basement membranes were fixed and stained with crystal violet.

In vitro wound healing assays. HeLa cells $\left(2 \times 10^{5}\right)$ were plated in $35-\mathrm{mm}$ cell culture dishes in $1.5 \mathrm{ml}$ of DMEM. When the cells grew to approximately $85 \%$ confluence, the wounds were made by scratching the monolayer culture using a sterile $200-\mu 1$ micropipette tip. The cells were then washed four times with $2 \mathrm{ml}$ of PBS (warmed to $37^{\circ} \mathrm{C}$ ) for $\sim 30 \mathrm{sec}$ per wash to remove any floating cells; $1.5 \mathrm{ml}$ of culture medium was then added. 
A

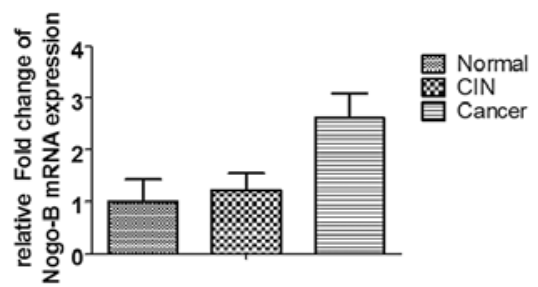

B

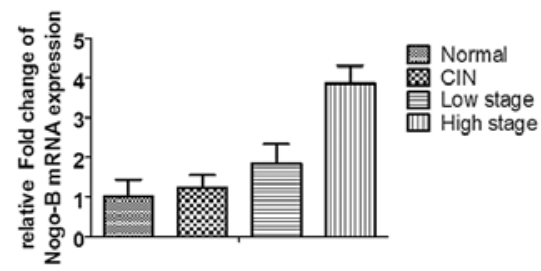

C

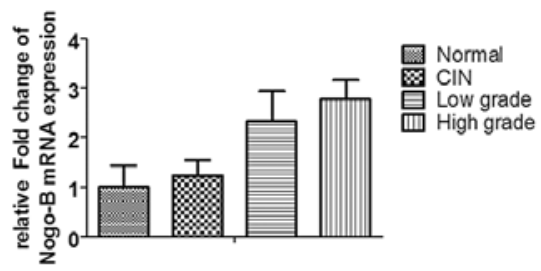

Figure 1. mRNA expression pattern of Nogo-B in cervical cancer. The Nogo-B mRNA expression in each tissue sample was relative to the GAPDH mRNA from the same sample. Nogo-B expression are plotted as mean \pm SD according to (A) Normal/Cancer, (B) tumor stages or (C) tumor grades.

Table II. Primer sets for real-time PCR.

\begin{tabular}{ll}
\hline Gene & \multicolumn{1}{c}{ Primer sequence } \\
\hline Nogo-B & F: 5'-GCAGTGTTGATGTGGGTATTT-3' \\
& R: 5'-CTGTGCCTGATGCCGTTC-3' \\
E-Cadherin & F: 5'-CAGGTCTCCTCATGGCTTTGC-3' \\
& R: 5'-CTTCCGAAAAGAAGGCTGTCC-3' \\
N-Cadherin & F: 5'-ATGCCCAAGACAAAGAAACC-3' \\
& R: 5'-CTGTGCTTGGCAAGTTGTCT-3' \\
Vimentin & F: 5'-GATGCGTGAGATGGAAGAGA-3' \\
& R: 5'-ATTTCAACGCCTCCAAGAAG-3' \\
Slug & F: 5'-ATTTCAACGCCTCCAAGAAG-3' \\
& R: 5'-CGAGGTGAGGATCTCTGGTT-3' \\
Snail & F: 5'-GAAGATGCACATCCGAAGC-3' \\
& R: 5'-GGAGAATGGCTTCTCACCAG-3' \\
TWIST1 & F: 5'-CGGACAAGCTGAGCAAGAT-3' \\
& R: 5'-GGACCTGGTACAGGAAGTCG-3' \\
ZEB1 & F: 5'-AACGGAAACCAGGATGAAAG-3' \\
& R: 5'-TTGTCACACAGGTCACATGC-3' \\
ZEB2 & F: 5'-CAAGTTCAAGTGCACGGAGT-3' \\
& R: 5'-GTTTGGGCATTCGTAAGGTT-3'
\end{tabular}

The wounded regions were marked on the outer bottom surface of the plate. Several selected fields $(n=4)$ from each wound and plate (time 0 ) were photographed using an inverted light microscope. The culture plates were placed back in the humidified cell incubator and incubated at $37^{\circ} \mathrm{C}, 5 \% \mathrm{CO}_{2}$ for up to $18-24 \mathrm{~h}$. The same wound fields from each plate were photographed every 6-8 h until the wound was completely closed.

Statistics. The data are presented as the mean \pm SD. SPSS 17.0 software (SPSS Inc., Chicago, IL, USA) was used for all statistical analyses with $\mathrm{P}<0.05$ considered statistically significant.

\section{Results}

Expression pattern of Nogo-B in cervical tissue samples. Samples including 14 normal cervical tissues, 10 cervical intraepithelial neoplasia (CIN) tissues, 31 cervical cancer tissues were analyzed by real-time PCR. Data were organized and presented as mean \pm SD according to tumor stage (T1-T2/ T3-T4) or grade (G1/G2-G3), and Nogo-B mRNA expression in each group were normalized to normal cervical tissue group (Fig. 1). We used one-way ANOVA statistics to analyse variance between normal tissues, CIN tissues and cancer tissues. The results showed that the expression of Nogo-B increased in cervical cancer (Fig. $1 \mathrm{~A}, \mathrm{P}=0.0438$ ), and the increased expression of Nogo-B was correlated with tumor stage (Fig. 1B, $\mathrm{P}=0.0005)$ and grade (Fig. $1 \mathrm{C}, \mathrm{P}=0.0109$ ).

Overexpression of Nogo-B inhibits HeL a cell proliferation. The function of a protein is always related to its distribution. Thus, we first determined the cellular localization of endogenous Nogo-B in HeLa cells using immunofluorescence. The results showed that Nogo-B, similar to other RTN family members, is mainly distributed in the cytoplasm and especially in the endoplasmic reticulum (ER) (Fig. 2A). In addition, we observed a low level of Nogo-B that was localized to the plasma membrane (PM) (Fig. 2A). Western blotting confirmed these observations; we detected endogenous Nogo-B expression both in the ER and on the PM (Fig. 2B).

To further investigate the effect of Nogo-B, we established several stable cell lines by overexpressing Nogo-B in HeLa cells (Fig. 2C). Among these, the NB-2 and NB-4 cell lines were selected to use in subsequent assays because they highly and moderately overexpressed Nogo-B, respectively, relative to the original HeLa cells.

During the establishment of Nogo-B stable transfectants, we had noted that the highly expressing Nogo-B HeLa cell lines grew more slowly with respect to the original HeLa cell line. Thus, we used the MTT assay to determine the proliferative rate of these HeLa cell lines. The results confirmed our observation: cells overexpressing Nogo-B had decreased cell proliferation (Fig. 2D). Duplicate proliferation assays demonstrated a similar effect. The average proliferation rates of NB-2 and NB-4 were only $1.64 \pm 0.15 \%$ and $1.57 \pm 0.08 \%$, respectively, which were both lower than the proliferation rate of Blank cells (1.87 \pm 0.21$)$.

Nogo-B promotes EMT in HeLa cells. We previously reported that Nogo-B could regulate cancer cell motility, and EMT has been described as a relevant process in tumor metastasis. Thus, we examined the effect of Nogo-B on EMT. We compared the cell adhesion, migration and invasion capabilities between the Blank, NB-4 and NB-2 HeLa cells, which had different 
A

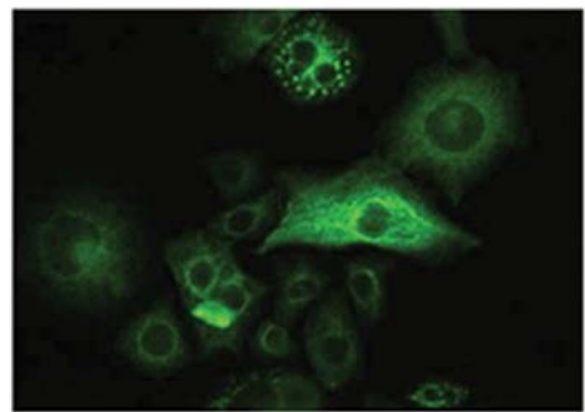

B

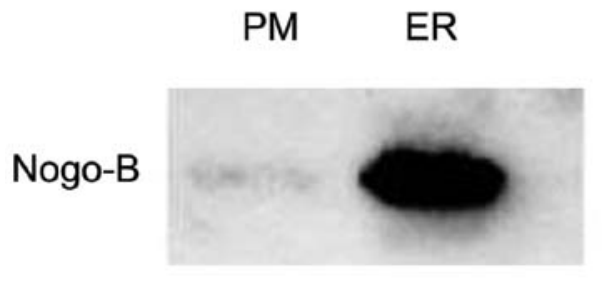

C

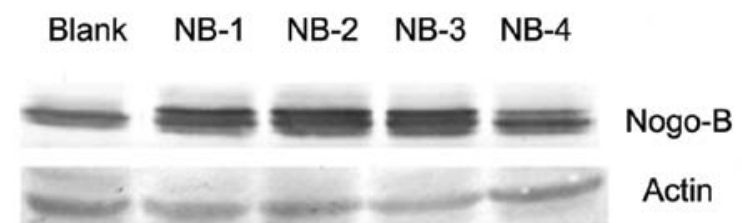

D

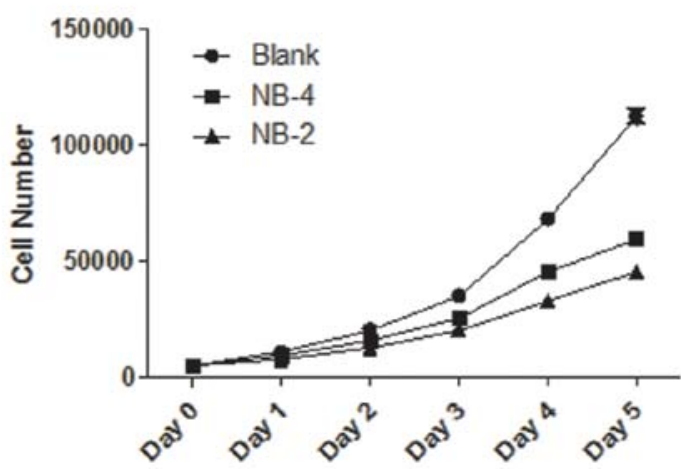

Figure 2. Nogo-B functions as a tumor suppressor gene in HeLa cells. (A) The distribution of endogenous Nogo-B in HeLa cells. HeLa cells were stained with a goat anti-Nogo-B antibody followed by a FITC anti-goat antibody (green) to visualize the localization of Nogo-B. The localization of endogenous Nogo-B was clearly observed in the ER and on the plasma membrane. (B) The components of the plasma membrane (PM) and endoplasmic reticulum (ER) were extracted from HeLa cells, and equivalent proteins were detected by western blotting. The results showed that Nogo-B was expressed mainly in the ER but also on the PM. (C) Western blotting of Nogo-B expression in Blank and different stably expressing Nogo-B cell lines. (D) The proliferation curves of Blank, NB-2 and NB-4 cells, which show that Nogo-B suppresses HeLa cell proliferation.

expression levels of the Nogo-B protein. The results showed that Nogo-B enhanced cell migration (Fig. 3B) and invasion (Fig. 3C) but inhibited the adhesion of HeLa cells (Fig. 3A) in a dose-dependent manner. This was especially true in the wound healing assay where we observed that HeLa cells had a random migration pattern (Fig. 3D), which has been described as novel mesenchymal behavior. Then, we analyzed the expression of EMT-related markers by western blotting. E-cadherin, an epithelial marker, was significantly downregulated in Nogo-B overexpressing cells compared with the empty vector-transfected cells. In contrast, the proteins $\mathrm{N}$-cadherin and vimentin, two mesenchymal markers, were upregulated in Nogo-B overexpressing cells compared with the control cells (Fig. 3E). Altogether, these data suggest that Nogo-B promotes EMT in HeLa cells.

Nogo-B and Fibulin-5 co-localize in pseudopods and enhance pseudopod formation. Due to the difference in cell behavior between the high and low Nogo-B overexpressing HeLa cells and the Blank HeLa cells, we investigated the other changes between the Nogo-B overexpressing cells and the Blank cells. We compared the morphological images of these three cell lines and found that the Nogo-B overexpressing cell lines formed more pseudopods than did the Blank cells. The number of pseudopod cells formed increased with the increase of Nogo-B expression (Fig. 4C).

Our previous study indicated that Nogo-B could interact with Fibulin-5 and increase Fibulin-5 secretion. Therefore, to further characterize the stimulation of Nogo-B overexpression on the behavior of HeLa cells, we performed a double immunostaining of Nogo-B and Fibulin-5 in the Nogo-B overexpressing cell line (NB-2), control cell line (Blank) and Blank cells transfected with SiA3 (a siRNA against Nogo-B, showed in Fig. 4B). The images of Nogo-B/Fibulin-5 staining revealed that, in NB-2 cells, Nogo-B and Fibulin-5 tended to accumulate in pseudopods (Fig. 4Ac1-e3). In contrast, the distribution of Nogo-B and Fibulin-5 in Blank cells was almost the same with a slight gathering around the nucleus (Fig. 4Ab1-b3). Additionally, in Blank cells transfected with $\mathrm{SiA} 3$, the ER that contained Nogo-B became porous (Fig. 4Aa1) and incomplete, and the cells became rounded. Moreover, the secretion of Fibulin-5 was inhibited, and Fibulin-5 accumulated in the cytoplasm (Fig. 4Aa2). We can also observe that the immunostaining of Nogo-B accumulated in different types of pseudopods, including filopodium (Fig. 4Ac1) and lamellipodium (Fig. 4Ad1). The enrichment of Nogo-B and Fibulin-5 in these pseudopods was much higher than that of the surrounding areas (blue arrow).

Of note, in Fig. 4Ae1-e3, we found that Nogo-B was present and that Fibulin-5 was absent from the tops of some pseudopods (white triangles), which may be evidence that Nogo-B functions as a carrier to bring Fibulin-5 to the membrane.

Downregulation of Fibulin-5 blocks EMT induced by Nogo-B. Since Fibulin-5 plays an important role in cell motility and is involved in cancer metastasis, we proposed that the EMT induced by overexpression of Nogo-B may be through its binding with Fibulin-5. Thus, we introduced a siRNA against Fibulin-5 (SiF5), which significantly downregulated the expression of the Fibulin-5 protein (Fig. 5A). Then, we examined the effect of SiF5 on EMT and cell motility in NB-2 cells that had 
A

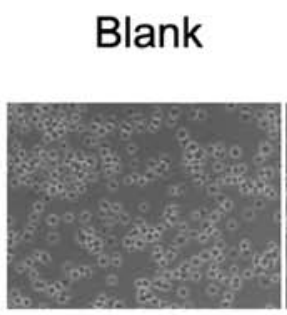

NB-4

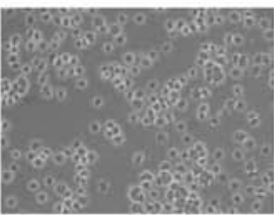

adhesion

B
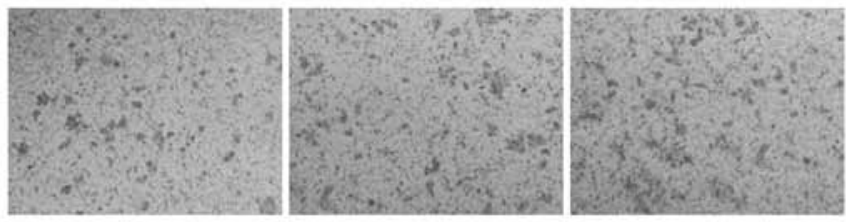

invasion

C
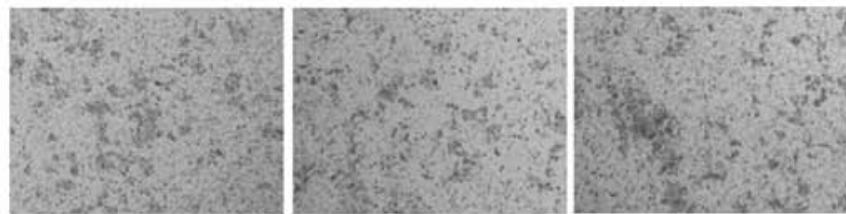

migration

D
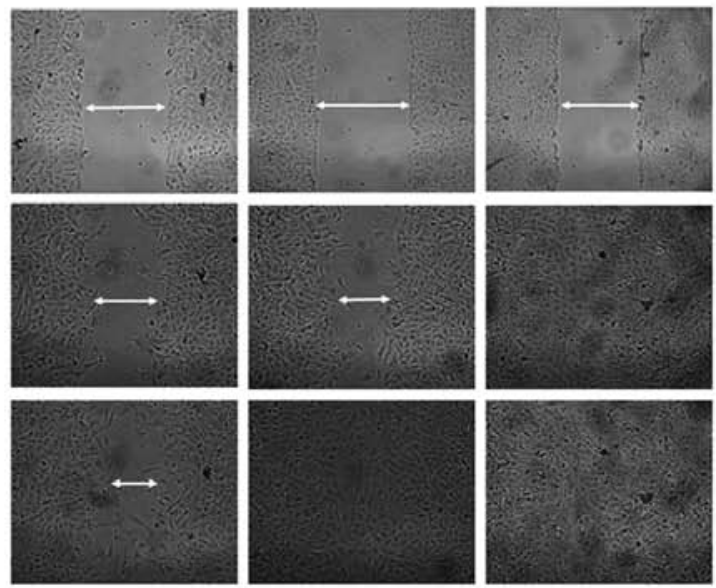

Oh

$24 \mathrm{~h}$

$48 \mathrm{~h}$
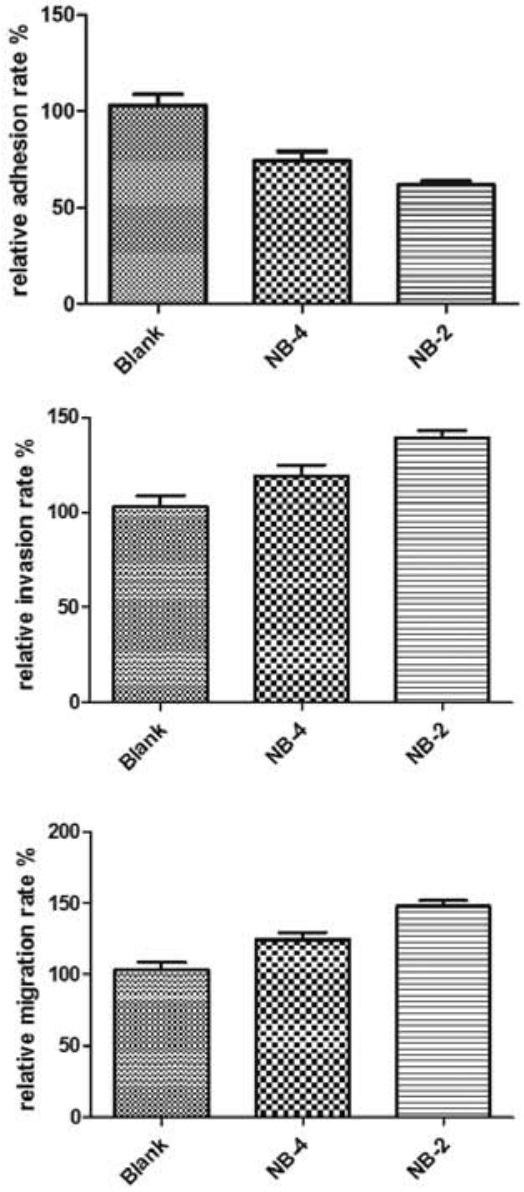

E

Blank NB-4 NB-2

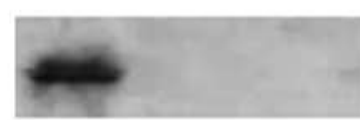

E-Cadherin

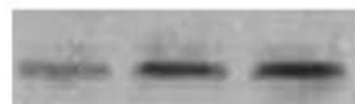

$\mathrm{N}$-Cadherin

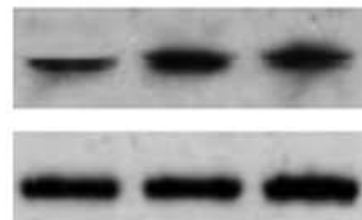

Vimentin

wound healing

Figure 3. Nogo-B promotes EMT in HeLa cells. The adhesion (A), migration (B) and invasion (C) of NB-2, NB-4 and Blank cells were examined by MTT or transwell assays. The left images show the experimental results from one experiment, while the right histogram shows three independent assays presented as the means \pm SE. The data are presented as percentages relative to Blank cells. (D) The in vitro wound healing assay exhibited different migration abilities of the NB-2, NB-4 and Blank cells. Additionally, the NB-2 and NB-4 cells also exhibited a random migration pattern, which has been described as a novel mesenchymal behavior. (E) Immunoblot analysis of epithelial (E-cadherin) and mesenchymal (N-cadherin and vimentin) marker proteins in NB-2, NB-4 and Blank cells.

the highest Nogo-B expression. As expected, compared with NB-2 cells transfected with a scrambled RNA, the migration and invasion levels of NB-2 cells transfected with SiF5 were downregulated, while the adhesion level was upregulated (Fig. 5D).
We also analyzed the mRNA and protein expression of EMT-related markers. The results showed that after SiF5 transfection, E-cadherin was visibly upregulated, while other mesenchymal markers, such as $\mathrm{N}$-cadherin and vimentin, or EMT inducers, such as Snail, TWIST1, ZEB1 and ZEB2, were 
A

\section{Blank+SiA3 Blank NB-2}
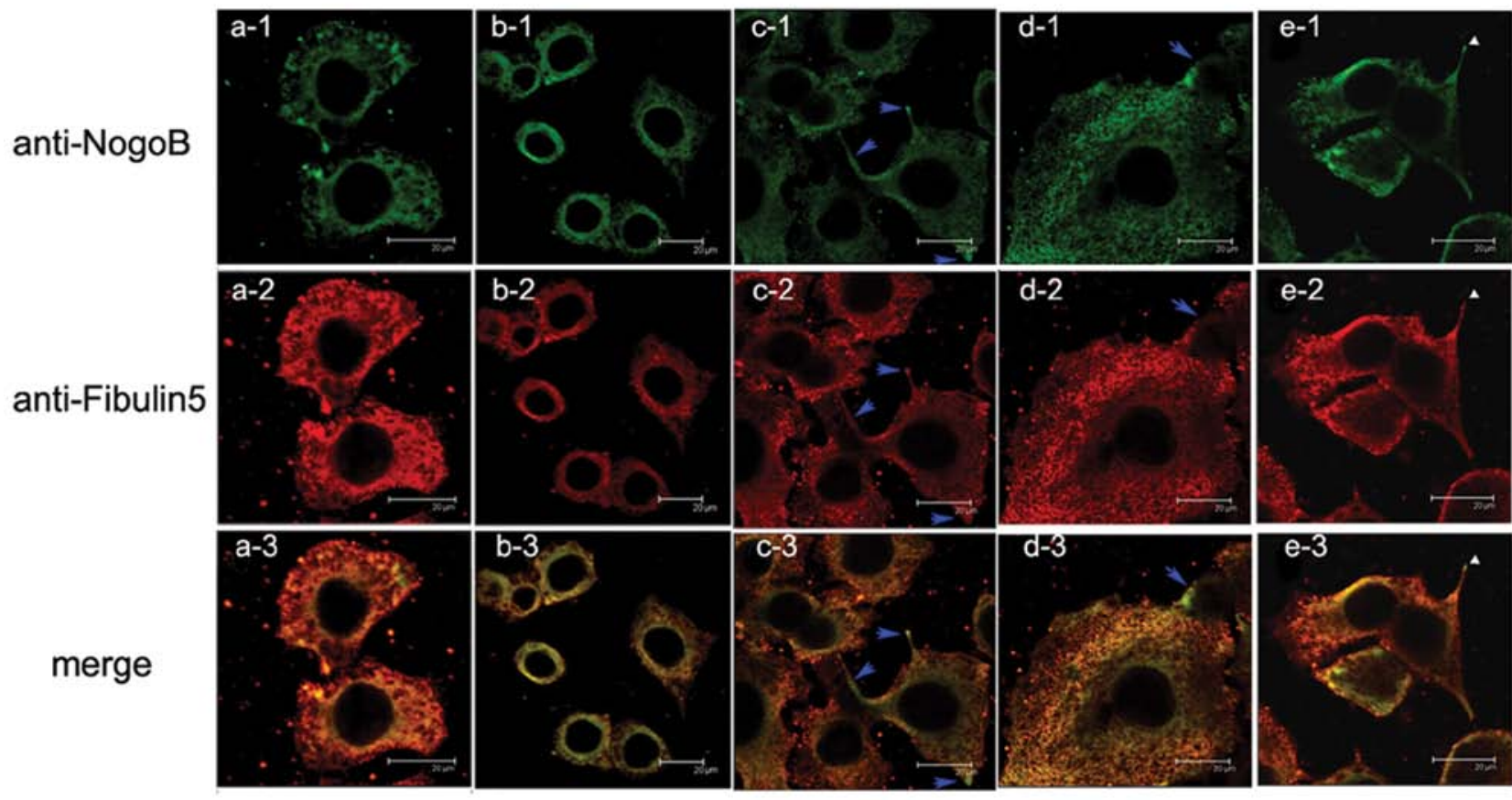

B

SiA1 SiA2 SiA3 SiNC
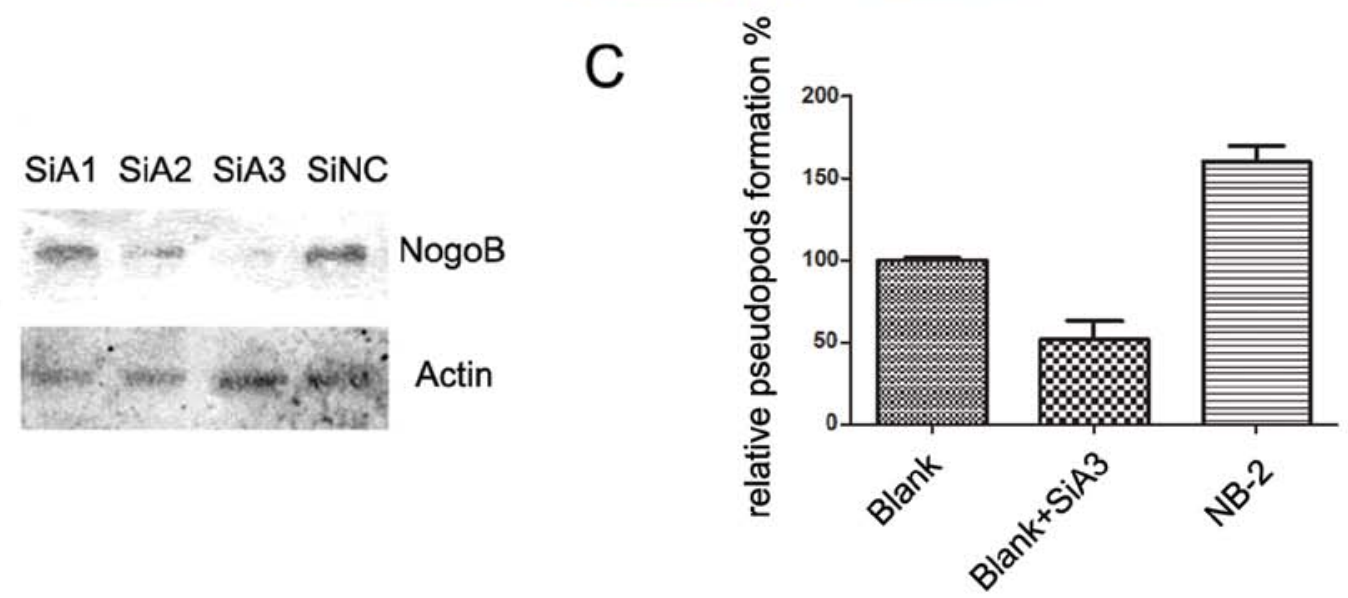

Figure 4. Nogo-B and Fibulin-5 co-localized in pseudopods and enhanced their formation. (A) The intracellular immunostaining of anti-Nogo-B and antiFBLN-5 antibodies in the Blank cell line transfected with the SiA3 plasmid, Blank cell line and NB-2 cell line. Nogo-B and Fibulin-5 tend to accumulate in pseudopods (blue arrows). Scale bars; $20 \mu \mathrm{m}$. (B) SiA3, a siRNA against Nogo-B, significantly reduced Nogo-B expression. (C) The expression of Nogo-B enhanced the formation of pseudopods. The data show five independent assays presented as the means $\pm \mathrm{SE}$ and as percentages relative to Blank cells.

downregulated. These results suggest that the EMT procedure induced by Nogo-B overexpression had been blocked by Fibulin-5 downregulation. Thus, Nogo-B may promote EMT through Fibulin-5.

\section{Discussion}

Uterine cervical cancer is one of the most common cancers in women worldwide, with an estimated global incidence of 470,000 new cases and approximately 233,000 deaths per year $(17,18)$. Cervical cancer is mainly contributed by the presence of high-risk human papillomavirus (HPV) oncogene expression (19). The viral protein products of HPV DNA interact with the anti-oncogenic function of the retinoblastoma and the p53 proteins and inactivate these tumor suppressors in normal keratinocytes. However, not all of those infected by
HPV develop cervical cancers; it indicates that factors other than HPV viral proteins also contribute to the progression to cervical cancer (20).

The process of EMT is essential for certain morphogenetic movements within the embryo and is strongly associated with the pathological process of tumor invasion $(3,4)$. During carcinoma invasion, EMT provides tumor cells with the ability to dissociate from one another and degrade and to actively migrate into the basal membrane and invade the adjacent connective tissues $(21,22)$. Cells undergoing EMT exhibit multiple phenotypic changes, such as the loss of apical-basal polarity, concomitantly with the acquisition of a motile behavior and a profound reorganization of the cytoskeleton $(23,24)$. Among these changes, the loss of expression or function of the E-cadherin cell-cell adhesion molecule has emerged as an important event for the local invasion of epithelial tumor cells, suggesting that 
A
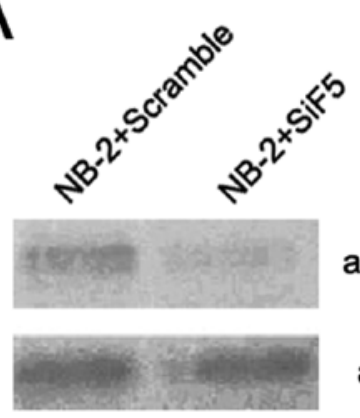

B

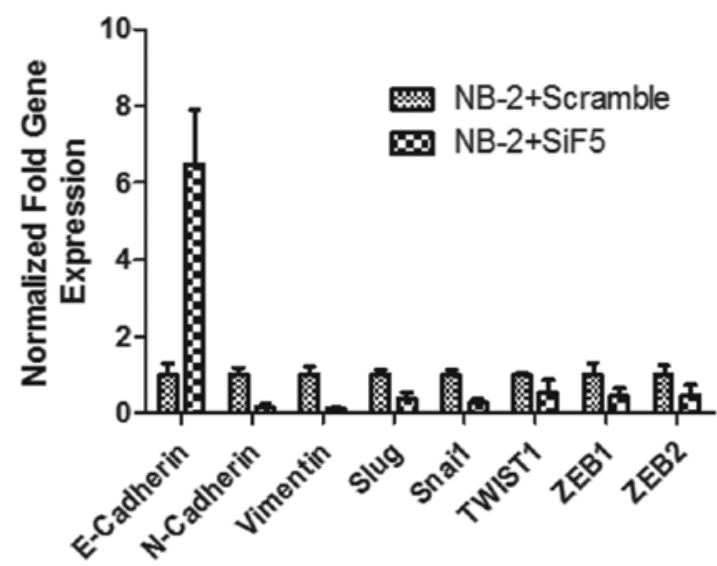

C

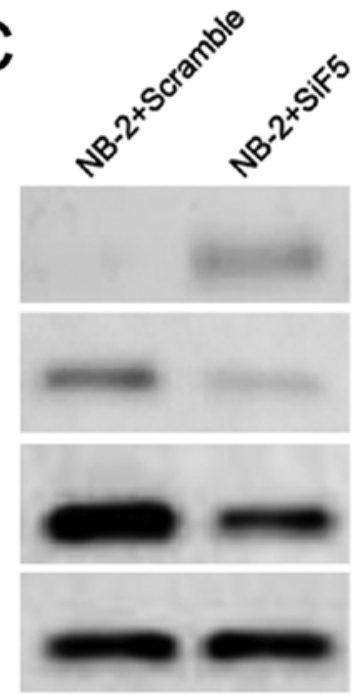

anti-Actin

anti-Fibulin5

E-Cadherin

$\mathrm{N}$-Cadherin

Vimentin

Actin

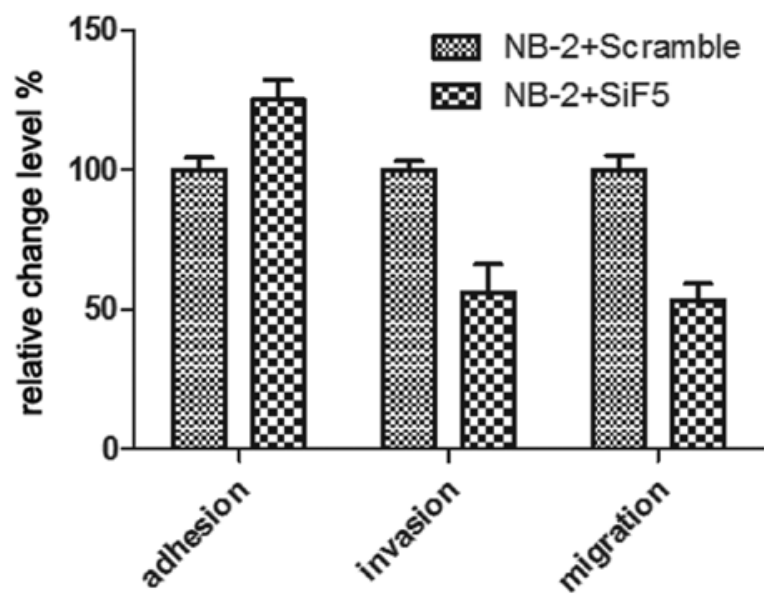

Figure 5. Downregulation of Fibulin-5 blocks EMT induced by Nogo-B. (A) Immunoblotting showed that SiF5 effectively inhibited Fibulin-5 expression. SiF5 reversed the effect of Nogo-B on EMT markers in both mRNA (B) and protein (C) levels. (D) SiF5 promoted NB-2 cell adhesion and inhibited cell migration and invasion. In all of the above assays, a scrambled small RNA was used as the negative control.

E-cadherin is an invasion-suppressor gene (25). Different growth factors and cytokines have also been implicated in the process of EMT in both epithelial cell systems and embryonic development, such as transforming growth factor- $\beta$ (TGF- $\beta$ ), which was first described as an inducer of EMT in normal mammary epithelial cells (MECs) (26) and is now recognized as a master regulator of EMT in a variety of cell types and tissues (27).

In this study, we first characterized that Nogo-B is associated with cervical cancer progression. Then we successfully identified Nogo-B as an inducer of EMT in HeLa cells. Overexpressing Nogo-B drastically downregulated the expression of E-cadherin while upregulating mesenchymal markers, such as $\mathrm{N}$-cadherin and vimentin. Furthermore, overexpression of Nogo-B could also inhibit cell adhesion while promoting cell migration and invasion. Nogo-B was originally identified as an apoptosis-inducing protein through multiple pathways $(8,9,28)$; recent studies on Nogo-B identified its function in cell motility. Nogo-B promotes vascular cell adhesion, stimulates endothelial cell migration and attenuates PDGF-induced smooth muscle migration (29). We previously found that Nogo-B mediates HeLa cell adhesion and motility (16). All of these results indicate that Nogo-B has multiple functions in different cells.

Pseudopods are cellular extensions used for movement. The direction and trajectory of cell movement depend on how the cells extend these pseudopods. In the absence of external or internal signals, pseudopods are not formed at random (30). Our observations showed that Nogo-B and Fibulin-5 preferred to accumulate in pseudopods, while the number of cells with pseudopods increased with an increase in Nogo-B expression.

Our previous studies showed that Fibulin-5 interacts with Nogo-B, while Fibulin-5 has also been reported to initiate and enhance epithelial-mesenchymal transition (EMT) and EMT induced by TGF- $\beta$ in mammary epithelial cells (31). Thus, we wondered whether Nogo-B promotes EMT via Fibulin-5. We introduced a siRNA that could effectively reduce the expression of Fibulin-5. These experiments showed that the EMT procedure induced by Nogo-B overexpression had been visibly blocked by the siRNA. Thus, our results suggested that Nogo-B may promote EMT partially through Fibulin-5. Although we cannot yet assess the complete signaling cascade of Nogo-B in EMT, our study may enrich the understanding of the pathways 
that regulate tumor cell movements and guide us to develop antagonists of metastasis as cervical cancer therapeutics.

\section{Acknowledgements}

This study was supported by National Natural Science Fund of China (No. 39880031), National Natural Science Fund key Project of China (No. C03031906), National 863 plans projects of China (No. 2007AA02Z156).

\section{References}

1. zur Hausen H: Human papillomaviruses in the pathogenesis of anogenital cancer. Virology 184: 9-13, 1991.

2. Lee MY, Chou CY, Tang MJ and Shen MR: Epithelialmesenchymal transition in cervical cancer: correlation with tumor progression, epidermal growth factor receptor overexpression, and snail up-regulation. Clin Cancer Res 14: 4743-4750, 2008.

3. Hay ED: An overview of epithelio-mesenchymal transformation. Acta Anat 154: 8-20, 1995 .

4. Thiery JP: Epithelial-mesenchymal transitions in tumour progression. Nat Rev Cancer 2: 442-454, 2002.

5. Yang J and Weinberg RA: Epithelial-mesenchymal transition: at the crossroads of development and tumor metastasis. Dev Cell 14: 818-829, 2008.

6. Oertle T, Merkler D and Schwab ME: Do cancer cells die because of Nogo-B? Oncogene 22: 1390-1399, 2003.

7. Su Z, Cao L, Zhu Y, et al: Nogo enhances the adhesion of olfactory ensheathing cells and inhibits their migration. J Cell Sci 120: 1877-1887, 2007.

8. Qi B, Qi Y, Watari A, et al: Pro-apoptotic ASY/Nogo-B protein associates with ASYIP. J Cell Physiol 196: 312-318, 2003.

9. Kuang E, Wan Q, Li X, Xu H, Zou T and Qi Y: ER stress triggers apoptosis induced by Nogo-B/ASY overexpression. Exp Cell Res 312: 1983-1988, 2006.

10. Liao H, Duka T, Teng FY, et al: Nogo-66 and myelin-associated glycoprotein (MAG) inhibit the adhesion and migration of Nogo-66 receptor expressing human glioma cells. J Neurochem 90: 1156-1162, 2004.

11. Miao RQ, Gao Y, Harrison KD, et al: Identification of a receptor necessary for Nogo-B stimulated chemotaxis and morphogenesis of endothelial cells. Proc Natl Acad Sci USA 103: 10997-11002, 2006.

12. Albig AR and Schiemann WP: Fibulin-5 function during tumorigenesis. Future Oncol 1: 23-35, 2005.

13. Nakamura T, Ruiz-Lozano P, Lindner V, et al: DANCE, a novel secreted RGD protein expressed in developing, atherosclerotic, and balloon-injured arteries. J Biol Chem 274: 22476-22483, 1999.
14. Nakamura T, Lozano PR, Ikeda Y, et al: Fibulin-5/DANCE is essential for elastogenesis in vivo. Nature 415: 171-175, 2002.

15. Albig AR, Neil JR and Schiemann WP: Fibulins 3 and 5 antagonize tumor angiogenesis in vivo. Cancer Res 66: 2621-2629, 2006.

16. Zhou S, Xiao W, Wan Q, et al: Nogo-B mediates HeLa cell adhesion and motility through binding of Fibulin-5. Biochem Biophys Res Commun 398: 247-253, 2010.

17. Matsukura T and Sugase M: Pitfalls in the epidemiologic classification of human papillomavirus types associated with cervical cancer using polymerase chain reaction: driver and passenger. Int J Gynecol Cancer 18: 1042-1050, 2008.

18. Munoz N, Bosch FX, de Sanjose S, et al: Epidemiologic classification of human papillomavirus types associated with cervical cancer. N Engl J Med 348: 518-527, 2003.

19. zur Hausen $\mathrm{H}$ : Papillomaviruses and cancer: from basic studies to clinical application. Nat Rev Cancer 2: 342-350, 2002.

20. Care A, Catalucci D, Felicetti F, et al: MicroRNA-133 controls cardiac hypertrophy. Nat Med 13: 613-618, 2007.

21. Liang X: EMT: new signals from the invasive front. Oral Oncol 47: 686-687, 2011.

22. Christofori G: New signals from the invasive front. Nature 441: 444-450, 2006.

23. Huber MA, Kraut $\mathrm{N}$ and Beug $\mathrm{H}$ : Molecular requirements for epithelial-mesenchymal transition during tumor progression. Curr Opin Cell Biol 17: 548-558, 2005.

24. Peinado H, Olmeda D and Cano A: Snail,Zeb and bHLH factors in tumour progression: an alliance against the epithelial phenotype? Nat Rev Cancer 7: 415-428, 2007.

25. Christofori G and Semb H: The role of the cell-adhesion molecule E-cadherin as a tumour-suppressor gene. Trends Biochem Sci 24: 73-76, 1999.

26. Miettinen PJ, Ebner R, Lopez AR and Derynck R: TGF-beta induced transdifferentiation of mammary epithelial cells to mesenchymal cells: involvement of type I receptors. J Cell Biol 127: 2021-2036, 1994.

27. Zavadil J and Bottinger EP: TGF-beta and epithelial-to-mesenchymal transitions. Oncogene 24: 5764-5774, 2005.

28. Li Q, Qi B, Oka K, et al: Link of a new type of apoptosis-inducing gene ASY/Nogo-B to human cancer. Oncogene 20: 3929-3936, 2001.

29. Acevedo L, Yu J, Erdjument-Bromage H, et al: A new role for Nogo as a regulator of vascular remodeling. Nat Med 10: 382-388, 2004.

30. Van Haastert PJ: How cells use pseudopods for persistent movement and navigation. Sci Signal 4: 6, 2011.

31. Lee YH, Albig AR, Regner M, Schiemann BJ and Schiemann WP: Fibulin-5 initiates epithelial-mesenchymal transition (EMT) and enhances EMT induced by TGF-beta in mammary epithelial cells via a MMP-dependent mechanism. Carcinogenesis 29: 2243-2251, 2008. 